\title{
Slot- versus round-shaped lateral windows for maxillary sinus floor augmentation: a 2-year clinical and radiographic study
}

\section{Abstract}
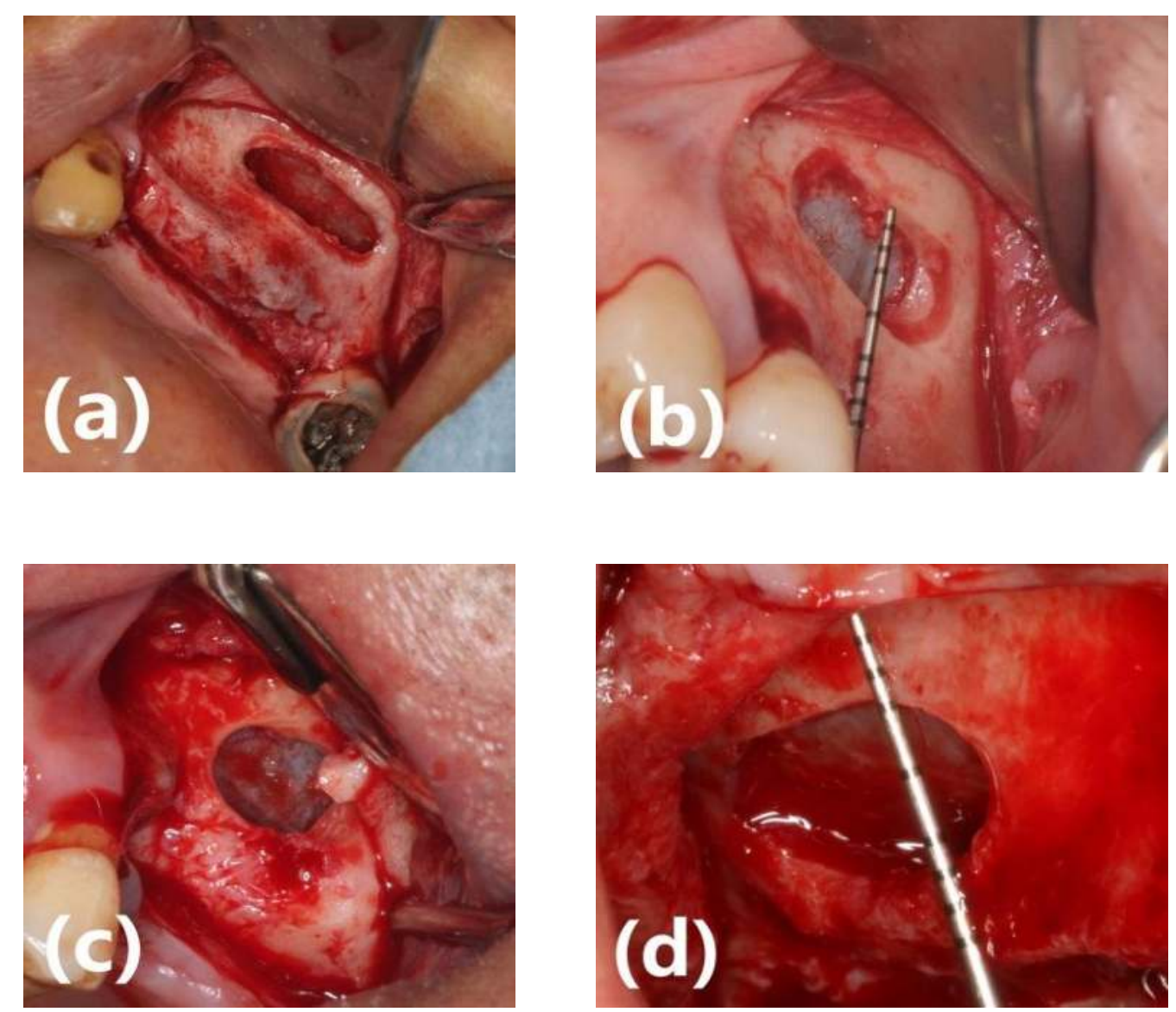

Lateral bone window size for sinus access. (a-b) Test group, slot-shaped the apicocoronal height was about 3-4 mm. (c-d) Control group, ovalshaped, the apicocoronal height was about 5-6 mm.

\section{Results}

A prominent augmented bone height $(\mathrm{ABH})$ was obtained in both groups (Test group: 10.53 $\pm 2.83 \mathrm{~mm}$, Control group: $9.23 \pm 3.24 \mathrm{~mm}$ ) with significant intergroup difference $(P=0.001)$, whereas no significant intergroup difference was found in augmented bone volume (ABV) $(P=0.359)$. Neither any significant intergroup difference emerged in data concerning the cumulative survival rate (CSR) nor the sinus membrane perforation rate. The augmented height loss $(\triangle A B H)$ of Test group was significantly lower than of Control group during the first 6 months $(P=0.020)$ and the second year $(P=0.037)$. Furthermore both $A B H$ and $A B V$ resorption rate of Test group was significantly lower than of Control group during the first 6 months after surgery $(P=0.022$, $\mathrm{P}=0.017)$.

\section{Conclusions}

The slot-shaped lateral technique is a simple and predictable surgica method for MSFA and simultaneous placement of dental implants. The technique facilitated to reduce apical augmented bone resorption during the first 6 months after surgery.

\section{Background and Aim}

- Very few clinical studies have investigated the influence of lateral window dimensions and appearance on the maturation and consolidation of graft in respect of maxillary sinus floor augmentation (MSFA). From a biological aspect, the residual bone wall represents a source of osteoblast cells; Mechanically, it helps the stability of blood clotting and protects the graft from possible mechanical stress. Previous experimental histological studies in animal have justified that bone formation and osteogenic process start from the sinus walls and septa, and then sprout into the elevated space along the implant surface, determining the maturation of central part of graft itself. Hence, we deemed approximate window dimensions and appearance may have an important influence on the maturation and consolidation of graft in the maxillary sinus.

\section{Methods and Materials}

Of 106 patients enrolled in the study, each underwent MSFA using deproteinized bovine bone mineral (DBBM) based on two different lateral window access flaps to maxillary sinus (Test group: slot-shaped access window; Control group: oval-shaped access window). Bone dimension and graft remodeling were measured at pre-op, immediately after the procedure (baseline) 6-month (T1), 1-year (T2) and 2-year (T3) post-op Cone-beam computed tomograph (CBCT) and panoramic radiographic imaging (OPT). CBCT scan data were analyzed with image processing software to evaluate the volume of grafted material throughout follow-up period. A life-table analysis was used to assess the implant survival. Intra-op and post-op complications were recorded.

\section{References}

1.Pjetursson, B E, W C Tan, M Zwahlen, et al., A systematic review of the success of sinus floor elevation and survival of implants inserted in combination with sinus floor elevation. Part I: Lateral approach. J Clin Periodontol, 2008. 35(8 Suppl): p. 216-40.

2. Hong, J Y, W S Baek, J K Cha, et al., Long-term evaluation of sinus floor elevation using a modified lateral approach in the posterior maxilla. Clin Oral Implants Res, 2016.

3. Jung, U W, J Y Hong, J S Lee, et al., A hybrid technique for sinus floor elevation in the severely resorbed posterior maxilla. Journal of Periodontal \& Implant Science, 2010. 40(2): p. 76-85.

4. Lin, I C, A M Gonzalez, H J Chang, et al., A 5-year follow-up of 80 implants in 44 patients placed immediately after the lateral trap-door window procedure to accomplish maxillary sinus elevation without bone grafting. Int J Oral Maxillofac Implants, 2011. 26(5): p. 1079-1086.

5.Borges, F L, R O Dias, A Piattelli, et al., Simultaneous sinus membrane elevation and dental implant placement without bone graft: a 6-month follow-up study. J Periodontol, 2011. 82(3): p. 403.

6. Avila-Ortiz, G, R Neiva, P Galindo-Moreno, et al., Analysis of the influence of residual alveolar bone height on sinus augmentation outcomes. Clin Oral Implants Res, 2012. 23(9): p. 1082-1088.

7.Dds, A D, M H D PhD, L S D PhD, et al., A Clinical and Histological Case Series Study on Calcium Sulfate for Maxillary Sinus Floor Augmentation and Delayed Placement of Dental Implants. Clin Implant Dent Relat Res, 2012. 14(2): p. 259-65.

8.Cordaro, L, D D Bosshardt, P Palattella, et al., Maxillary sinus grafting with Bio-Oss $\AA^{\circ}$; or Straumann ${ }^{\circledR}$; Bone Ceramic: histomorphometric results from a randomized controlled multicenter clinical trial. Clin Ora Implants Res, 2008. 19(8): p. 796-803.

9.Consultant, A M D, A 10-Year Clinical and Radiographic Study of Implants Placed after Maxillary Sinus Floor Augmentation with an 80:20 Mixture of Deproteinized Bovine Bone and Autogenous Bone. Clin Implan Dent Relat Res, 2012. 16(3): p. 435-446.

10.Hatano, N, Y Shimizu and K Ooya, A clinical long-term radiographic evaluation of graft height changes after maxillary sinus floor augmentation with a 2:1 autogenous bone/xenograft mixture and simultaneous placement of dental implants. Clin Oral Implants Res, 2004. 15(3): p. 33945. 